\title{
Quatre espèces nouvelles d'Endodontidae (Mollusca, Pulmonata) éteints de Rurutu (Îles Australes, Polynésie française)
}

\author{
Gabrielle ZIMMERMANN \\ Muséum national d'Histoire naturelle, \\ Département Milieux et Peuplements aquatiques, \\ case postale 26, 57 rue Cuvier, F-75231 Paris cedex 05 (France) \\ zimmermann@mnhn.fr \\ Olivier GARGOMINY \\ Benoît FONTAINE
} Muséum national d'Histoire naturelle, Département Systématique et Évolution, case postale 51, 57 rue Cuvier, F-75231 Paris cedex 05 (France)

gargo@mnhn.fr fontaine@mnhn.fr

MOTS CLÉS Mollusca, Gastropoda, Endodontidae, mollusques terrestres, extinction,

Polynésie française, espèces nouvelles.

KEY WORDS Mollusca, Gastropoda, Endodontidae, land snails, extinction,

French Polynesia, new species.
Zimmermann G., Gargominy O. \& Fontaine B. 2009. - Quatre espèces nouvelles d'Endodontidae (Mollusca, Pulmonata) éteints de Rurutu (Îles Australes, Polynésie française). Zoosystema 31 (4) : 791-805.

\section{RÉSUMÉ}

Quatre espèces d'escargots terrestres Endodontidae (Mollusca, Pulmonata) sont décrites, toutes endémiques de l'île de Rurutu (Îles Australes, Polynésie française): Australdonta collicella n. sp., A. magnasulcatissima n. sp., A. microspiralis n. sp. et $A$. sulcata n. sp. Le nombre d'espèces d'Endodontidae connu pour Rurutu est porté de sept à 11 . Toutes sont considérées comme éteintes sur l'île, seules des coquilles vides ont pu être récoltées.

\section{ABSTRACT}

Four new extinct species of Endodontidae (Mollusca, Pulmonata) from Rurutu (Austral Islands, French Polynesia).

Four species of land snails belonging to the family Endodontidae (Mollusca, Pulmonata) are described, all of them are endemic to Rurutu Island (Austral Islands, French Polynesia): Australdonta collicella n. sp, A. magnasulcatissima n. sp., A. microspiralis n. sp. and A. sulcata n. sp. The number of species of Endodontidae known from Rurutu is increased from seven to 11 . All of them are considered extinct. 


\section{INTRODUCTION}

La faune de Gastéropodes terrestres des îles du Pacifique est connue pour sa richesse, ses forts taux d'endémisme insulaires et sa grande vulnérabilité face à la dégradation anthropique des habitats (Cowie 1992, 2001a, b; Hadfield et al. 1993; Abdou \& Bouchet 2000; Bouchet \& Abdou 2003; Ohbayashi et al. 2007). Ce phénomène, connu depuis 100 ans (Preece 1998), est aujourd'hui extrêmement alarmant et fait des Gastéropodes terrestres l'un des groupes les plus menacés au monde (Lydeard et al. 2004).

Parmi les 13 majeures familles de Gastéropodes pulmonés du Pacifique (Pilsbry 1916; Cowie 1996), les Achatinellidae Gulick, 1873 et les Endodontidae Pilsbry, 1895 sont remarquables par l'importance de leur radiation (Solem 1976, 1981 ; Hadfield 1986).

L'archipel des Australes est un archipel isolé, situé à l'extrême sud de la Polynésie française. La famille des Endodontidae y est remarquable pour sa grande radiation, avec 41 espèces autochtones inventoriées par Solem $(1976,1981)$, presque toutes endémiques de l'une ou l'autre des îles de l'archipel.

Rurutu, la plus septentrionale des Îles Australes, attire l'attention par la présence de massifs soulevés calcaires carbonatés (Clouard \& Bonneville 2003) à la périphérie de l'île volcanique et par la très forte érosion de son couvert végétal (Hallé 1983). Deux espèces d'Endodontidae y furent décrites au XIXe siècle par Garrett (1879), mais les Endodontidae de Rurutu furent véritablement collectés pour la première fois par la Mangarevan Expedition du Bernice Pauahi Bishop Museum d'Honolulu (1934) dans le cadre de ses récoltes malacologiques aux Australes (Cooke 1935), par Baker (1940) ainsi que par Cooke \& Kondo (1961). Ce fut néanmoins Solem $(1976,1981)$ qui se pencha en détail sur les spécimens d'Entodontidae récoltés par la Mangarevan Expedition. Cinq nouvelles espèces furent alors décrites, portant à sept le nombre d'espèces connues sur l'île. Elles furent réparties en deux genres: Minidonta Solem, 1976 (une espèce, endémique de Rurutu: Minidonta haplaenopla Solem, 1976) et Australdonta Solem, 1976 (six taxons: A. degagei (Garrett, 1879); A. magnasulcata
Solem, 1976; A. radiella rurutuensis (Garrett, 1879); A. pseudplanulata Solem, 1976; A. tapina Solem, 1976; A. yoshii Solem, 1976; les cinq derniers sont endémiques de Rurutu).

Un abondant matériel a été récolté en 2003 à Rurutu lors d'une mission pluridisciplinaire d'inventaire de la biodiversité des Îles Australes inscrite au Contrat de Développement État-Territoire de Polynésie française 2000-2003.

Toutes les espèces inventoriées par Solem (1976, 1981) sur la base du matériel récolté par la Mangarevan Expedition ont été retrouvées ainsi que d'autres, nouvelles pour la science, dont quatre sont ici décrites, portant le nombre d'espèces d'Endodontidae connues pour Rurutu de sept à 11 .

L'intégralité de ce matériel est constituée de coquilles vides, aucun spécimen n'ayant été récolté vivant sur l'île depuis le XIXe siècle. La famille des Endodontidae est très probablement éteinte sur Rurutu, et son absence contraste aujourd'hui avec l'abondance d'espèces allochtones.

Ces résultats sont discutés en insistant sur l'importance d'identifier les espèces éteintes pour qu'un bilan des extinctions de Gastéropodes terrestres du Pacifique puisse être dressé et pour que des mesures de conservation de leurs habitats puissent être prises.

\section{MATÉRIEL ET MÉTHODES}

Les prospections ont été effectuées à Rurutu du 17 au 30 novembre 2003. Soixante-neuf stations ont été prospectées, en particulier dans les "matos", récifs soulevés très riches en mollusques. En particulier, des récoltes ont été effectuées dans des zones d'accumulation de litière où se concentrent les coquilles. Les stations déjà visitées par la Mangarevan Expedition ont été prospectées, y compris les localités types des espèces décrites par Solem $(1976,1981)$.

Deux types d'échantillonnage ont été effectués pour chaque station: récolte à vue pour les espèces les plus grandes et tamisage de litière et de sol pour échantillonner les espèces mesurant moins de $5 \mathrm{~mm}$, très majoritaires. Le premier tamisage (mailles de $1 \mathrm{~cm}$ environ) a été effectué sur place, à l'aide d'un tamis Winkler. Le résidu a ensuite été séché puis 


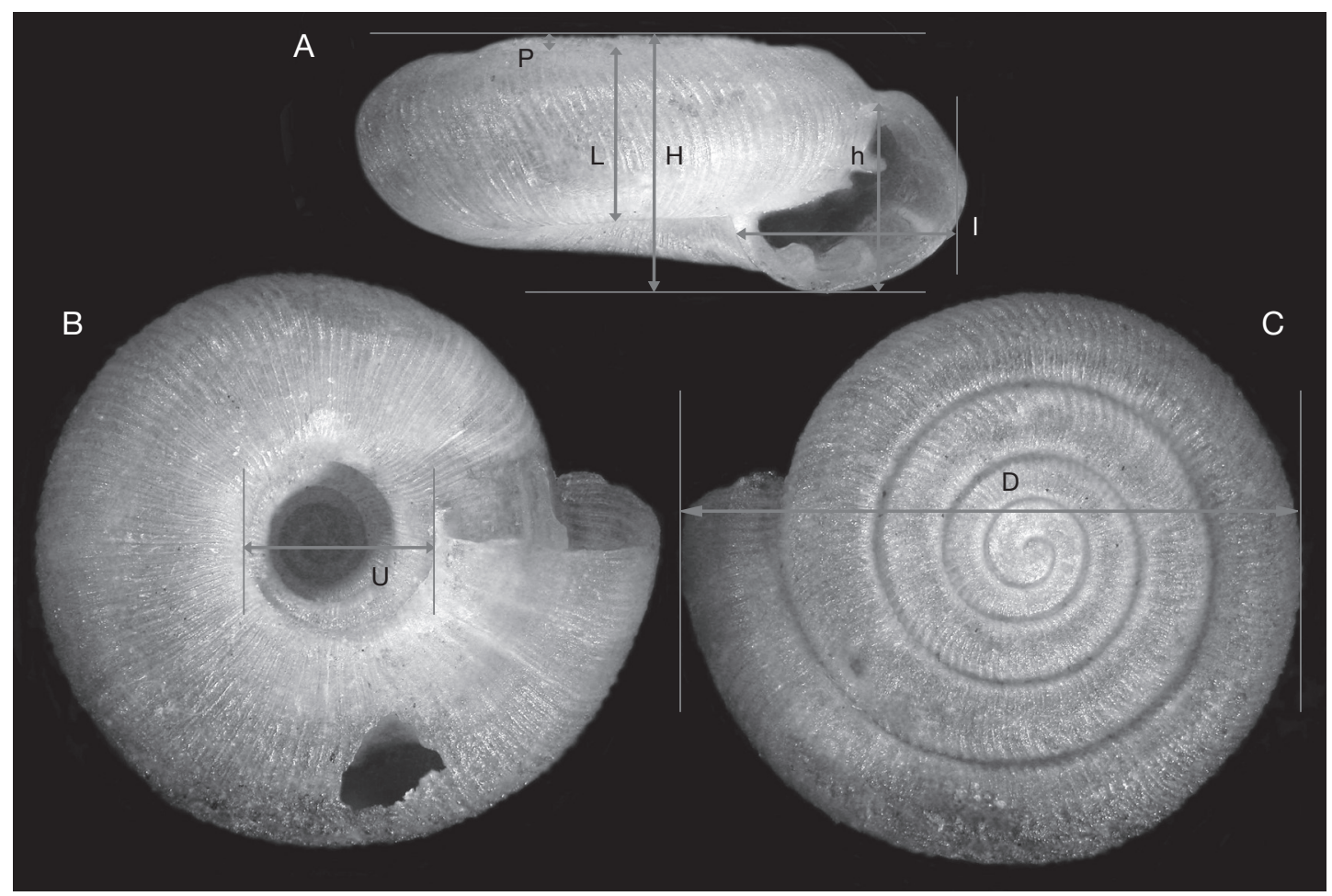

FIG. 1. - Plans de prise de vue et mesures standardisées utilisées pour la description formelle des Endodontidae (spécimen: Australdonta pseudplanulata Solem, 1976, holotype [BPBM 148204], $D=2.86 \mathrm{~mm}$ ): A, vue frontale; B, face inférieure; C, face supérieure. Abréviations: D, diamètre de la coquille; $\mathbf{h}$, hauteur de l'ouverture; $\mathbf{H}$, hauteur de la coquille; I, largeur de l'ouverture; L, hauteur du dernier tour de spire; $\mathbf{P}$, élévation de la spire; $\mathbf{U}$, diamètre de l'ombilic.

tamisé avec des mailles de plus en plus petites (3, 2 et $0,63 \mathrm{~mm})$. Les fractions de taille supérieure à $2 \mathrm{~mm}$ ont été triées à l'œil nu, les plus petites à la loupe binoculaire. La fraction inférieure à $0,63 \mathrm{~mm}$ n’a pas été conservée. Au total 14991 coquilles d'Endodontidae ont été collectées.

Les espèces d'Endodontidae sont classiquement définies sur des critères anatomiques (essentiellement relatifs à l'appareil reproducteur) et conchyliologiques (Solem 1976). Seuls ces derniers ont pu être examinés puisqu'aucun animal vivant n'a été collecté.

\section{TERMinOlogie}

La terminologie utilisée est celle établie par Solem (1976). Hauteur de la coquille (H), diamètre de la coquille (D), diamètre de l'ombilic (U), hauteur du dernier tour de spire (L), élévation de la spire $(\mathrm{P})$, largeur de l'ouverture (l) et hauteur de l'ouverture (h). Les mesures (Fig. 1) sont données en mm. Pour chaque mesure, sont données: la valeur relevée sur l'holotype, la moyenne des valeurs observées sur l'ensemble des spécimens et l'intervalle des valeurs minimales et maximales. Les barrières pariétales sont comptées à partir de la plus abaxiale en allant vers la columelle et sont notées $\operatorname{Pr}$ (Fig. 2). Les barrières palatales sont comptées à partir de la plus adaxiale en s'éloignant de la columelle et sont notées P. Les barrières columellaires sont comptées à partir de la plus adaxiale en s'éloignant de la columelle, sont notées $\mathrm{C}$, mais sont le plus souvent réduites en nombre ou perdues chez les Endodontidae.

\section{ABRÉVIATIONS}

BPBM Bernice Pauahi Bishop Museum, Honolulu; MNHN Muséum national d'Histoire naturelle, Paris; MEB microscopie électronique à balayage. 


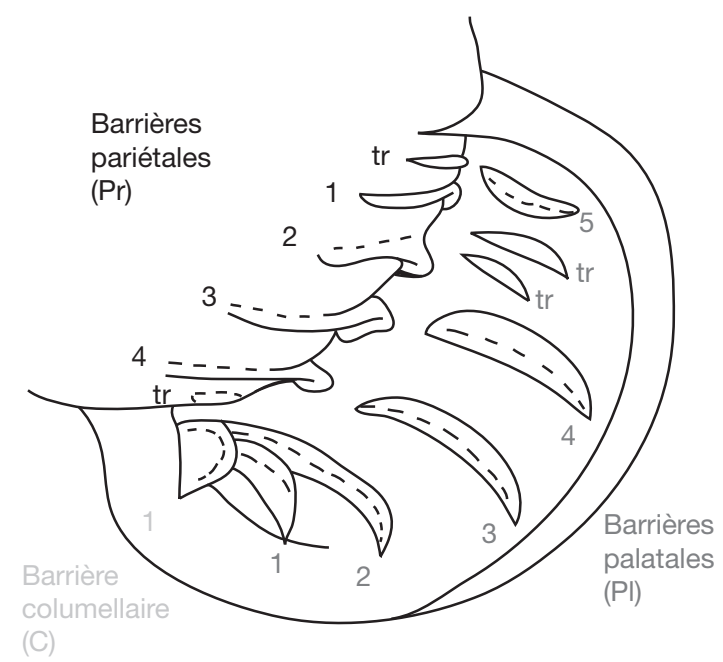

FIG. 2. - Terminologie en vigueur pour la désignation des barrières aperturales des Endodontidae, d'après Solem (1976).

\section{SYSTÉMATIQUE}

Ordre PULMONATA Cuvier, 1814 Sous-ordre STYLOMMATOPHORA Schmidt, 1855

Superfamille PUnCTOIDEA Morse, 1864

Famille Endodontidae Pilsbry, 1895

Genre Australdonta Solem, 1976

Australdonta Solem, 1976: 289.

ESPÈCE TYPE. - Australdonta raivavaeana Solem, 1976 par désignation originale.

Distribution. - Endémique de l'archipel des Australes.

\section{Australdonta collicella n. sp.}

(Figs 3A; 4)

Matériel type. - Holotype (MNHN 20952), 8 paratypes (MNHN 20953), 1 paratype (BPBM 271800).

LOCALITÉ TYPe. - Rurutu, sud du Mato de Peva, falaise de Toerau, pied de falaise avec blocs rocheux, Barringtonia, Hibiscus, Pandanus, calcaire, $22,47058^{\circ} \mathrm{S}, 151,32522^{\circ} \mathrm{W}$, 15 m, exposition SW, 21.XI.2003, coll. Gargominy \& Fontaine, stn Rr31.
ÉTYMOLOGIE. - Du latin collicellus (petite colline), en raison de son aspect de dôme conféré par une suture très peu profonde.

MATÉRIEL EXAMINÉ. - 296 spécimens, coll. Gargominy \& Fontaine dont holotype et 8 paratypes (MNHN), 1 paratype (BPBM).

Stn Rr04, sommet du Mato Arei, étroit canyon calcaire avec Hibiscus, Pandanus, Asplenium et forêt avec cocotiers, $22,45646^{\circ} \mathrm{S}, 151,32684^{\circ} \mathrm{W}, 95 \mathrm{~m}, 17 . \mathrm{XI} .2003,43$ spécimens. - Stn Rr05, Mato Arei, pied de falaise calcaire, Hibiscus, Pandanus, Asplenium, 22,45607 ${ }^{\circ} \mathrm{S}, 151,32778^{\circ} \mathrm{W}$, 0 m, exposition SW, 17.XI.2003, 2 spécimens. - Stn Rr08, côté $S$ du Mato Arei, $\mathrm{N}$ de Peva Iti, pied de falaise calcaire, Barringtonia, $22,46402^{\circ} \mathrm{S}, 151,32479^{\circ} \mathrm{W}, 15 \mathrm{~m}$, exposition SSE, 17.XI.2003, 16 spécimens. - Stn Rr19, S Itirama, Mato S d'Hauti, pied de blocs calcaires, Hibiscus, Pandanus, Aleurites, $22,49583^{\circ} \mathrm{S}, 151,32506^{\circ} \mathrm{W}, 80 \mathrm{~m}$, exposition S, 19.XI.2003, 2 spécimens. - Stn Rr20, Paparai, S Hauti, W Anatataura, bas de falaise calcaire, Musa, Macropiper, Aleurites, $22,50075^{\circ} \mathrm{S}, 151,32813^{\circ} \mathrm{W}, 10 \mathrm{~m}$, exposition SW, 19.XI.2003, 3 spécimens. - Stn Rr22, falaise côtière calcaire, $S$ d'Hauti, N Pointe Poroa, pente rocheuse avec Pandanus, $22,49311^{\circ} \mathrm{S}, 151,32392^{\circ} \mathrm{W}, 20 \mathrm{~m}$, exposition NE, 19.XI.2003, 3 spécimens. - Stn Rr31, 61 spécimens (dont holotype et tous les paratypes). - Stn Rr38, sortie nord du village d'Hauti, pied du Mato Toarutu, pente rocheuse à Hibiscus avec Citrus, 22,48491 ${ }^{\circ} \mathrm{S}, 151,32625^{\circ} \mathrm{W}$, 5 m, exposition S, 22.XI.2003, 1 spécimen. - Stn Rr39, Mato Toarutu, versant W, S de Pointe Mauo, pente rocheuse volcanique à Barringtonia et Pandanus, sous grande grotte, $22,48062^{\circ} \mathrm{S}, 151,3227^{\circ} \mathrm{W}, 15 \mathrm{~m}$, exposition E, 22.XI.2003, 5 spécimens. - Stn Rr40, S Paparai, versant E de la vallée, zone d'accumulation en pied de falaise calcaire à Hibiscus, Pandanus et Elaeocarpus, 22,50378 ${ }^{\circ} \mathrm{S}, 151,33164^{\circ} \mathrm{W}, 15 \mathrm{~m}$, exposition W, 22.XI.2003, 6 spécimens. - Stn Rr46a, Pointe Arei, bord de route, gangue solide fossile dans le calcaire, entre deux planchers stalagmitiques, $22,45489^{\circ} \mathrm{S}$, $151,32446^{\circ} \mathrm{W}, 10 \mathrm{~m}, 23 . \mathrm{XI} .2003$, 1 spécimen. - Stn Rr46b, Pointe Arei, bord de route, terre fossilifère sous gangue dans le calcaire, $22,45489^{\circ} \mathrm{S}, 151,32446^{\circ} \mathrm{W}, 10 \mathrm{~m}$, 23.XI.2003, 10 spécimens. - Stn Rr52, S Paparai, E de la route, fond de vallon en forêt à Hibiscus et Inocarpus, caféiers en sous-bois, $22,51247^{\circ} \mathrm{S}, 151,33572^{\circ} \mathrm{W}, 80 \mathrm{~m}$, exposition E, 25.XI.2003, 1 spécimen. - Stn Rr66, E Mato Toarutu, blocs rocheux en pied de falaise calcaire, au dessus plantation, Hibiscus, $22,47973^{\circ} \mathrm{S}, 151,32449^{\circ} \mathrm{W}$, 30 m, exposition NW, 28.XI.2003, 61 spécimens. — Stn Rr68, N Mato Toarutu, grotte calcaire, zone traditionnelle de récolte d'escargots, zone d'accumulation entre falaise et petit pic, $22,47749^{\circ} \mathrm{S}, 151,32232^{\circ} \mathrm{W}, 45 \mathrm{~m}, 28 . \mathrm{XI} .2003,81$ spécimens - Stn Rr69, Peva, Maperevaru, rochers en pied de falaise calcaire, Hibiscus, $22,47415^{\circ} \mathrm{S}, 151,32291^{\circ} \mathrm{W}$, 10 m, exposition E, 28.XI.2003, 1 spécimen.

Distribution. - Endémique insulaire: Rurutu, sur la côte Est, entre les pointes Arei et Toataratara. 


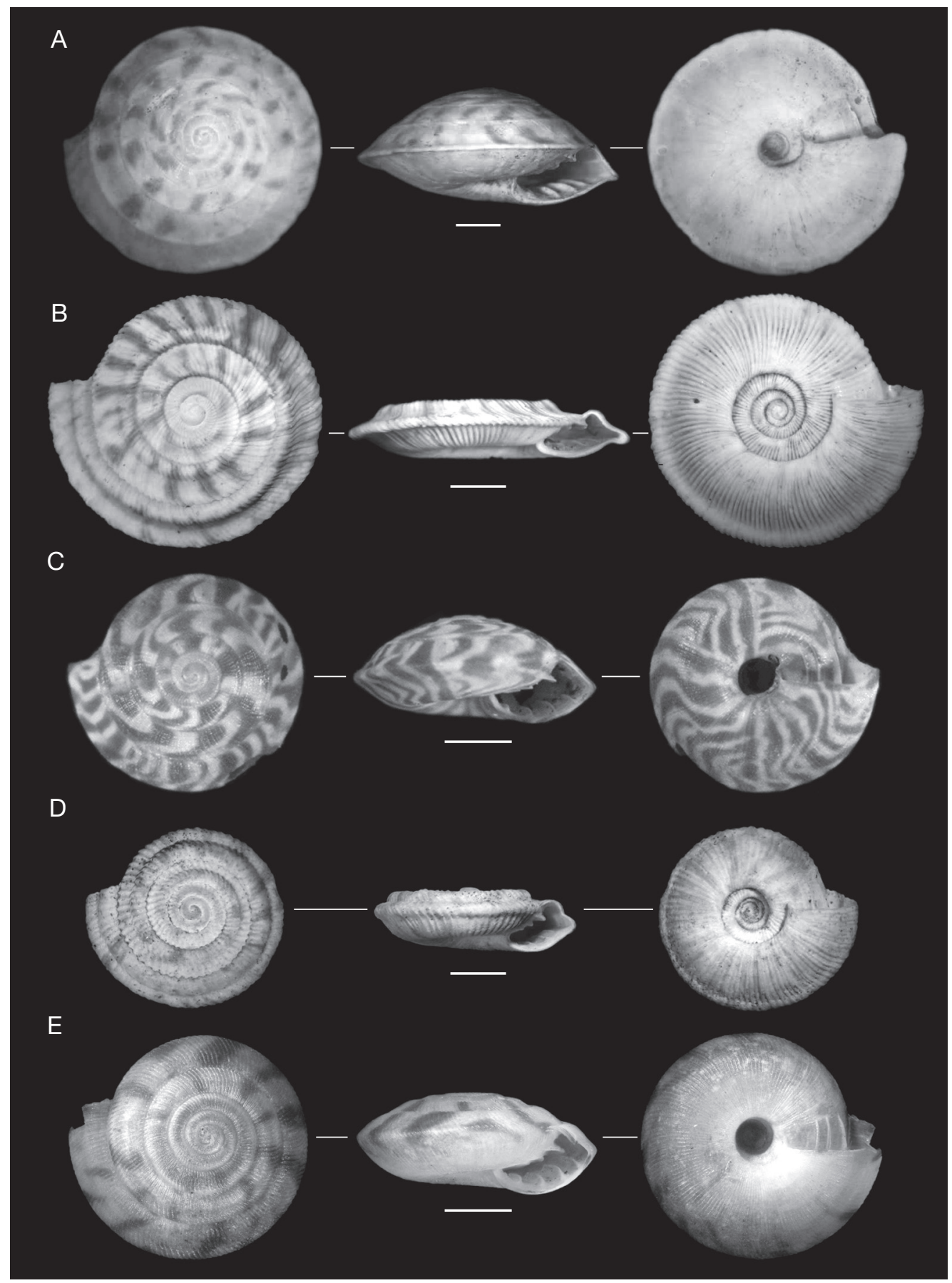

FIG. 3. - Holotypes d'Endodontidae de Rurutu, îles Australes, Polynésie française (D, diamètre de la coquille): A, Australdonta collicella n. sp. (MNHN 20952), D =5,80 mm; B, A. magnasulcatissima n. sp. (MNHN 20954), D =5,04 mm; C, Australdonta microspiralis n. sp. (MNHN 20956), D = 3,50 mm; D, A. sulcata n.sp. (MNHN 20958), D =3,59 mm; E, A. tapina Solem, 1976 (BPBM 148838a), $\mathrm{D}=3,56 \mathrm{~mm}$. Échelles: $1 \mathrm{~mm}$. 


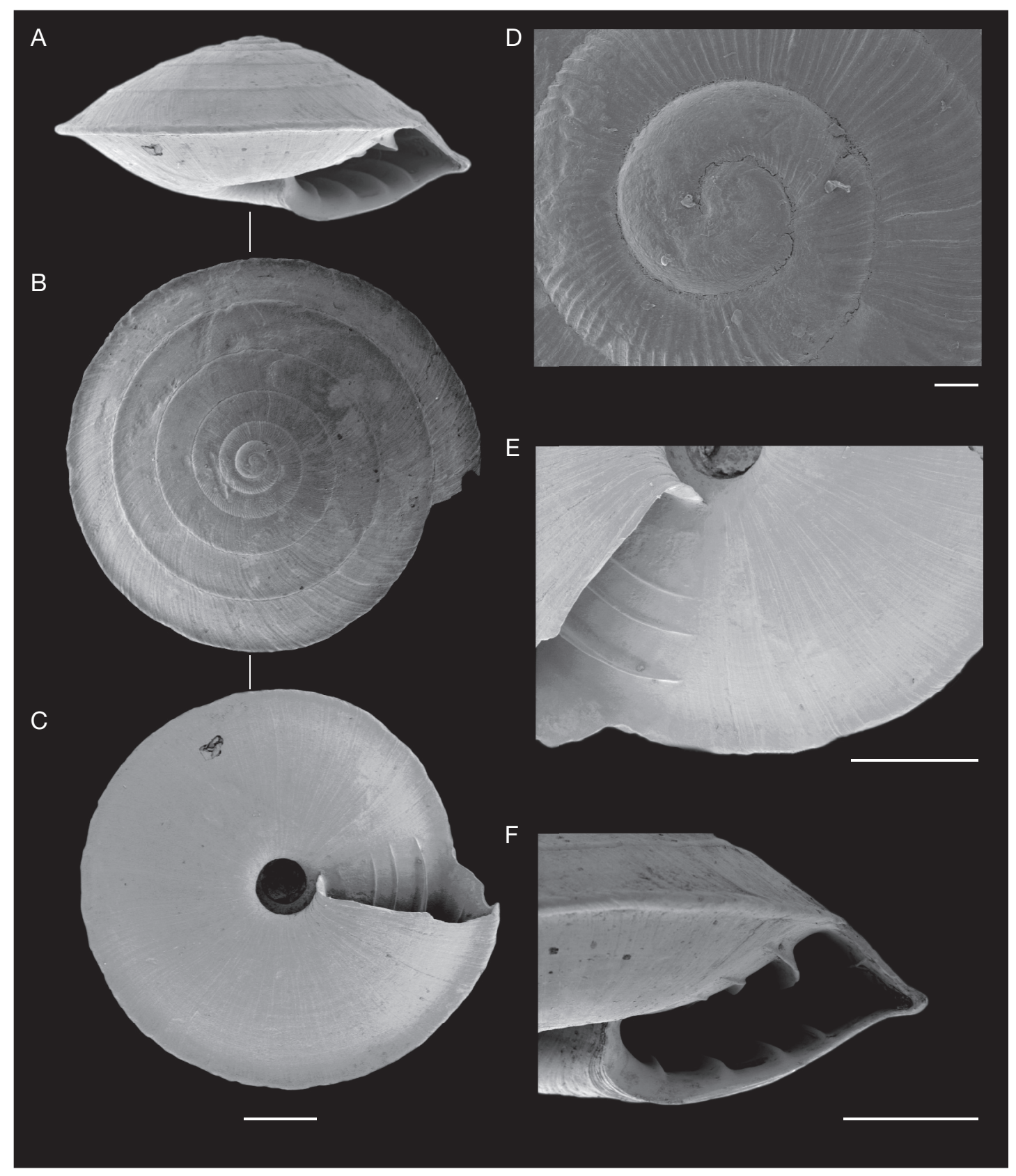

FIG. 4. - Australdonta collicella n. sp., Rurutu, Îles Australes, Polynésie française (MEB): A, vue frontale; B, face supérieure; C, face inférieure; D, détail de la protoconque; E, sculpture du dernier tour, face inférieure; F, détail de l'ouverture. Échelles: A-C, E, F, 1 mm; D, 100 mm.

\section{DESCRIPTION (HOLOTYPE)}

Coquille presque discoïde, très carénée, à spire modérément élevée, formée de six tours et deux tiers séparés par une suture presque invisible, bordée par un faible bourrelet. Couleur blanc crème ornée de flammules orangées. Protoconque d'un tour 
TABleau 1. - Dimensions (en mm) des paratypes d'Australdonta collicella n. sp., A. magnasulcatissima n. sp., A. microspiralis n. sp. et A. sulcata n. sp. Abréviations: $\mathbf{D}$, diamètre de la coquille; $\mathbf{h}$, hauteur de l'ouverture; $\mathbf{H}$, hauteur de la coquille; $\mathbf{I}$, largeur de l'ouverture; L, hauteur du dernier tour de spire; P, élévation de la spire; Stn, Station de récolte; U, diamètre de l'ombilic.

\begin{tabular}{|c|c|c|c|c|c|c|c|c|c|}
\hline Espèce & Spécimen & Stn & $\mathbf{H}$ & I & h & $\mathbf{P}$ & $\mathbf{L}$ & D & $\mathbf{U}$ \\
\hline \multirow[t]{9}{*}{ Australdonta collicella n. sp. } & paratype 1 MNHN 20953 & Rr31 & 3,19 & 2,32 & 1,45 & 1,04 & 1,51 & 6,61 & 1,09 \\
\hline & paratype 2 MNHN 20953 & Rr31 & 2,70 & 2,35 & 1,16 & 0,80 & 1,30 & 6,01 & 0,92 \\
\hline & paratype 3 MNHN 20953 & Rr31 & 2,80 & 2,41 & 1,31 & 0,88 & 1,33 & 5,90 & 0,95 \\
\hline & paratype 4 MNHN 20953 & Rr31 & 2,80 & 2,28 & 1,12 & 0,78 & 1,42 & 6,00 & 0,90 \\
\hline & paratype 5 MNHN 20953 & Rr31 & 2,66 & 2,15 & 1,20 & 0,80 & 1,26 & 5,63 & 1,10 \\
\hline & paratype 6 MNHN 20953 & Rr31 & 2,99 & 2,58 & 1,31 & 0,88 & 1,51 & 6,13 & 0,85 \\
\hline & paratype 7 MNHN 20953 & Rr31 & 3,01 & 2,31 & 1,22 & 0,96 & 1,41 & 6,05 & 1,05 \\
\hline & paratype 8 MNHN 20953 & Rr31 & 2,71 & 2,14 & 1,13 & 0,86 & 1,29 & 5,68 & 1,18 \\
\hline & paratype 9 BPBM 271800 & Rr31 & 3,04 & 2,25 & 1,26 & 0,84 & 1,49 & 6,03 & 1,09 \\
\hline \multirow[t]{5}{*}{ A. magnasulcatissima $\mathrm{n} . \mathrm{sp}$. } & paratype 1 MNHN 20955 & Rr31 & 1,14 & 1,52 & 0,81 & 0,00 & 0,87 & 4,83 & 1,73 \\
\hline & paratype 2 MNHN 20955 & Rr31 & 1,21 & 1,40 & 0,79 & 0,00 & 0,94 & 4,69 & 1,74 \\
\hline & paratype 3 MNHN 20955 & Rr31 & 1,18 & 1,36 & 0,78 & 0,00 & 0,84 & 5,02 & 1,99 \\
\hline & paratype 4 MNHN 20955 & Rr31 & 1,00 & 1,41 & 0,82 & 0,00 & 0,78 & 4,37 & 1,42 \\
\hline & paratype 5 BPBM 271801 & Rr31 & 1,11 & 1,45 & 0,78 & 0,00 & 0,77 & 4,48 & 1,57 \\
\hline \multirow[t]{9}{*}{ A. microspiralis $\mathrm{n} . \mathrm{sp}$. } & paratype 1 MNHN 20957 & Rr53 & 1,60 & 1,31 & 0,87 & 0,20 & 0,91 & 3,34 & 0,61 \\
\hline & paratype 2 MNHN 20957 & $\operatorname{Rr} 53$ & 1,65 & 1,47 & 0,95 & 0,20 & 0,98 & 3,62 & 0,59 \\
\hline & paratype 3 MNHN 20957 & Rr53 & 1,83 & 1,44 & 0,97 & 0,32 & 0,99 & 3,58 & 0,50 \\
\hline & paratype 4 MNHN 20957 & Rr53 & 1,52 & 1,27 & 0,81 & 0,31 & 0,99 & 3,12 & 0,42 \\
\hline & paratype 5 MNHN 20957 & Rr53 & 1,77 & 1,41 & 0,90 & 0,39 & 0,92 & 3,59 & 0,57 \\
\hline & paratype 6 MNHN 20957 & $\operatorname{Rr} 53$ & 1,54 & 1,34 & 0,84 & 0,36 & 0,94 & 3,31 & 0,51 \\
\hline & paratype 7 MNHN 20957 & Rr53 & 1,79 & 1,38 & 0,83 & 0,38 & 0,93 & 3,49 & 0,51 \\
\hline & paratype 8 MNHN 20957 & Rr53 & 1,83 & 1,40 & 0,94 & 0,38 & 0,96 & 3,38 & 0,53 \\
\hline & paratype 9 BPBM 271802 & $\operatorname{Rr} 53$ & 1,65 & 1,35 & 0,94 & 0,27 & 0,92 & 3,35 & 0,51 \\
\hline \multirow[t]{4}{*}{ A. sulcata n. sp. } & paratype 1 MNHN 20959-20960 & Rr22 & 1,19 & 1,09 & 0,73 & 0,00 & 0,83 & 3,73 & 1,45 \\
\hline & paratype 2 MNHN 20959-20960 & Rr22 & 0,96 & 1,04 & 0,74 & 0,00 & 0,74 & 3,36 & 1,10 \\
\hline & paratype 3 MNHN 20959-20960 & Rr39 & 1,18 & 1,04 & 0,73 & 0,00 & 0,80 & 3,05 & 0,97 \\
\hline & paratype 4 BPBM 271803 & Rr39 & 1,18 & 1,00 & 0,76 & 0,00 & 0,84 & 3,17 & 0,98 \\
\hline
\end{tabular}

environ, lisse; transition protoconque/téléoconque indistincte. La téléoconque ne porte pas de côtes primaires. L'ensemble de la coquille porte des costules radiales très serrées, croisant des microstries spirales très fines. Le réseau résultant forme des mailles rectangulaires horizontales. Ombilic caréné, en forme de $\mathrm{U}$ très étroit occupant $17 \%$ du diamètre au niveau du dernier tour. Bord columellaire subcaréné. Ouverture en croissant de lune dévié par la carène; péristome prosocline, non continu, formant un angle évasé à la base du bord columellaire. Barrières aperturales: quatre lamelles pariétales, cinq lamelles palatales, la quatrième étant plus basse que les autres et la cinquième étant au dessus de la carène, pas de columellaire.

\section{DimENSIONS}

Dimensions de l'holotype: $\mathrm{H}=2,78 \mathrm{~mm} ; \mathrm{D}=$ $5,80 \mathrm{~mm} ; \mathrm{U}=1,01 \mathrm{~mm} ; \mathrm{L}=1,42 \mathrm{~mm} ; \mathrm{P}=0,71 \mathrm{~mm}$; $\mathrm{l}=2,25 \mathrm{~mm} ; \mathrm{h}=1,25 \mathrm{~mm} ; \mathrm{D} / \mathrm{U}=5,74 ; \mathrm{H} / \mathrm{D}=$ 0,$48 ; \mathrm{h} / \mathrm{l}=0,55$.

Dimensions moyennes et écarts-types pour 29 individus adultes (holotype, paratypes et matériel d'étude) : $\mathrm{H}=2,64 \mathrm{~mm} \pm 0,25 ; \mathrm{D}=5,64 \mathrm{~mm} \pm$ 0,$41 ; \mathrm{U}=0,88 \mathrm{~mm} \pm 0,14 ; \mathrm{L}=1,32 \mathrm{~mm} \pm 0,12$; $\mathrm{P}=0,77 \mathrm{~mm} \pm 0,12 ; 1=2,29 \mathrm{~mm} \pm 0,12 ; \mathrm{h}=$ $1,20 \mathrm{~mm} \pm 0,11 ; \mathrm{D} / \mathrm{U}=6,48 \pm 0,79 ; \mathrm{H} / \mathrm{D}=$ $0,47 \pm 0,02 ; \mathrm{h} / \mathrm{l}=0,52 \pm 0,04$.

Les dimensions des paratypes sont données dans le Tableau 1.

\section{Remarques}

Le nombre des lamelles pariétales varie de 1 à 4 parmi les individus observés. La face inférieure de la coquille peut être plus ou moins convexe (très convexe chez les jeunes). Cette espèce se distingue par une grande taille pour le genre (plus de $5 \mathrm{~mm}$ de diamètre). 


\section{Australdonta magnasulcatissima n. sp.} (Figs 3B; 5)

MATÉRIEL TYPE. - Holotype (MNHN 20954), 4 paratypes (MNHN 20955), 1 paratype (BPBM 271801).

LOCALITÉ TYPe. - Rurutu, sud du Mato de Peva, falaise de Toerau, pied de falaise avec blocs rocheux, Barringtonia, Hibiscus, Pandanus, calcaire, $22,47058^{\circ} \mathrm{S}, 151,32522^{\circ} \mathrm{W}$, 15 m, exposition SW, 21.XI.2003, coll. Gargominy \& Fontaine, stn $\operatorname{Rr} 31$.

ÉTYMOLOGIE. - Cette espèce ressemble à une Australdonta magnasulcata Solem, 1976 très grande, très plane et très carénée. Le suffixe latin -issimus (au plus haut degré) est donc ajouté.

Matériel eXAminé. - Stn Rr31, 17 spécimens, coll. Gargominy \& Fontaine dont holotype et 4 paratypes (MNHN), 1 paratype (BPBM).

Distribution. - Endémique insulaire: Rurutu; uniquement connue du Mato Toerau.

\section{DESCRIPTION (HOLOTYPE)}

Coquille presque discoïde et carénée, à spire très basse, formée de quatre tours et trois quarts séparés par une suture bien marquée. La spire est plane, l’apex est invisible en vue frontale. Couleur blanc crème ornée de flammules fauves visibles principalement sur la partie supérieure de la coquille. La spire porte un bourrelet adapical. Protoconque d'un tour environ, avec des traces de côtes radiales. Transition protoconque/téléoconque indistincte. Sculpture de la téléoconque constituée de côtes primaires épaisses mais peu élevées, au nombre de 92 sur le dernier tour de spire, espacées de 125-135 $\mu$ m et légèrement inclinées dans le sens contraire à l'accroissement, donnant un aspect d' "écailles». Microsculpture de costules radiales secondaires très serrées (25 à 30 entre deux côtes primaires), couvrant toute la surface de la coquille, y compris au niveau des côtes primaires, et croisant des microstries spirales très fines et très nombreuses. Le réseau en résultant est de maille carrée. Ombilic très large, à enroulement spiral, occupant $38 \%$ du diamètre du dernier tour. Bord columellaire sub-caréné. Ouverture en croissant de lune dévié par la carène; péristome légèrement prosocline, non continu, formant un angle évasé à la base du bord columellaire. Barrières aperturales: quatre lamelles pariétales, la pariétale supérieure (Pr1) étant la plus forte; cinq lamelles palatales, la cinquième étant au dessus de la carène; pas de columellaire.

\section{DiMENSIONS}

Dimensions de l'holotype: $\mathrm{H}=1,10 \mathrm{~mm} ; \mathrm{D}=$ $5,04 \mathrm{~mm} ; \mathrm{U}=1,92 \mathrm{~mm} ; \mathrm{L}=0,88 \mathrm{~mm} ; \mathrm{P}=$ $0,00 \mathrm{~mm} ; \mathrm{l}=1,48 \mathrm{~mm} ; \mathrm{h}=0,78 \mathrm{~mm} ; \mathrm{D} / \mathrm{U}=$ 2,$63 ; \mathrm{H} / \mathrm{D}=0,22 ; \mathrm{h} / \mathrm{l}=0,53$.

Dimensions moyennes et écarts-types pour 9 individus adultes (holotype, paratypes et matériel d'étude) : $\mathrm{H}=1,07 \mathrm{~mm} \pm 0,09 ; \mathrm{D}=4,59 \mathrm{~mm} \pm$ 0,$32 ; \mathrm{U}=1,63 \mathrm{~mm} \pm 0,22 ; \mathrm{L}=0,83 \mathrm{~mm} \pm 0,06$; $\mathrm{P}=0,00 \mathrm{~mm} \pm 0,00 ; \mathrm{l}=1,43 \mathrm{~mm} \pm 0,05 ; \mathrm{h}=$ $0,79 \mathrm{~mm} \pm 0,03 ; \mathrm{D} / \mathrm{U}=2,84 \pm 0,19 ; \mathrm{H} / \mathrm{D}=$ $0,23 \pm 0,01 ; \mathrm{h} / \mathrm{l}=0,56 \pm 0,03$.

Les dimensions des paratypes sont données dans le Tableau 1.

\section{REMARQUES}

La plupart des individus ne possèdent que trois barrières pariétales. Cette espèce se distingue par une grande taille pour le genre (plus de $5 \mathrm{~mm}$ de diamètre). Elle est syntopique avec Australdonta magnasulcata Solem, 1976. Du fait de leur récolte au niveau de zones d'accumulation de coquilles vides, cette syntopie pourrait néanmoins être un artefact. La présence du bourrelet adapical est un caractère partagé par plusieurs espèces de Rurutu (A. magnasulcata Solem, 1976, A. magnasulcatissima n. sp. et $A$. sulcata n. sp.), et est toujours accompagnée d'un épaississement des côtes.

\section{Australdonta microspiralis n. sp.}

$$
\text { (Figs 3C; 6) }
$$

MATÉRIEL TYPE. - Holotype (MNHN 20956), 8 paratypes (MNHN 20957), 1 paratype (BPBM 271802).

LOCALITÉ TYPE. - Rurutu, côte sud-ouest, environ $1 \mathrm{~km}$ de la Pointe Toataratara, est de la route, pied de falaise, plantation (Musa, taros), calcaire, $22,51851^{\circ} \mathrm{S}$, $151,34696^{\circ} \mathrm{W}, 40 \mathrm{~m}$, exposition WSW, 25.XI.2003, coll. Gargominy \& Fontaine, stn Rr53.

ÉTYMOLOGIE. - En raison de la présence caractéristique de stries spirales fines aussi marquées que les côtes radiales, cette espèce est nommée à partir du préfixe micro- (très petit) suivi du terme latin spiralis (spiralé). 


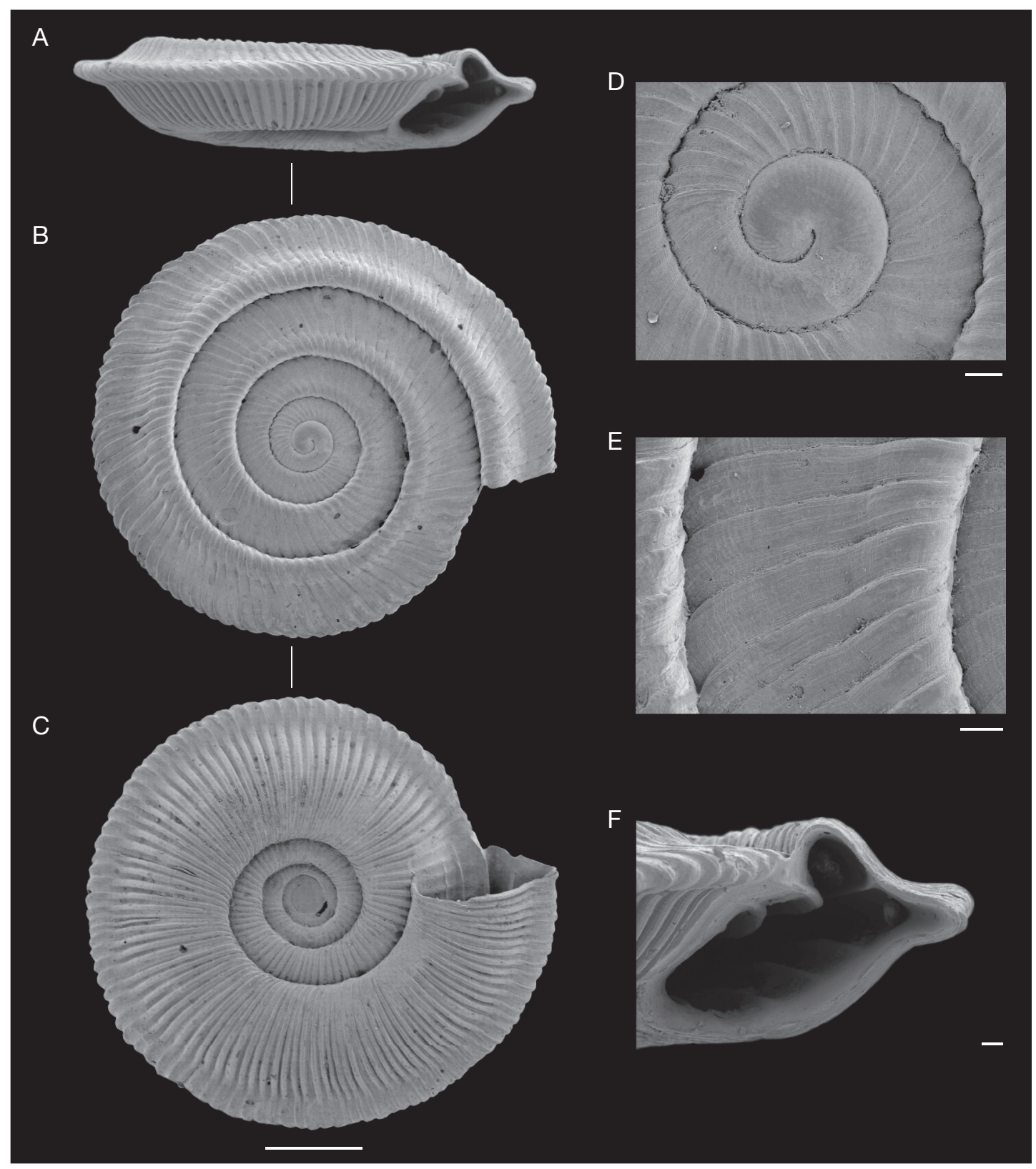

FIG. 5. - Australdonta magnasulcatissima n. sp., Rurutu, îles Australes, Polynésie française (MEB): A, vue frontale; B, face supérieure; $\mathbf{C}$, face inférieure; $\mathbf{D}$, détail de la protoconque; E, sculpture et microsculpture de l'avant dernier tour; F, détail de l'ouverture. Échelles: A-C, $1 \mathrm{~mm} ; \mathrm{D}-\mathrm{F}, 100 \mu \mathrm{m}$.

MATÉRIEL EXAMINÉ. — 114 spécimens, coll. Gargominy \& Fontaine dont holotype et 8 paratypes (MNHN), 1 paratype (BPBM).

Stn Rr35, S Paparai, versant E de la vallée, rochers dans pente calcaire, Barringtonia, $22,50796^{\circ} \mathrm{S}, 151,33548^{\circ} \mathrm{W}$, $30 \mathrm{~m}$ exposition E, 22.XI.2003, 29 spécimens. - Stn Rr40, S Paparai, versant E de la vallée, zone d'accumulation au pied de falaise calcaire à Hibiscus, Pandanus et 
Elaeocarpus, $22,50378^{\circ} \mathrm{S}, 151,33164^{\circ} \mathrm{W}, 15 \mathrm{~m}$, exposition W, 22.XI.2003, 2 spécimens. - Stn Rr53, 81 spécimens (dont l'holotype et tous les paratypes). - Stn Rr54, côte SE, $1 \mathrm{~km}$ NE Te Araroa, pied de falaise calcaire, Hibiscus et Barringtonia, 22,50811 ${ }^{\circ} \mathrm{S}, 151,3286^{\circ} \mathrm{W}, 10 \mathrm{~m}$, exposition E, 25.XI.2003, 3 spécimens.

DistRiBution. - Endémique insulaire: Rurutu, sur la côte est de l'île, dans les environs de Paparai et Naairoa.

\section{DESCRIPTION (HOLOTYPE)}

Coquille presque discoïde, carénée, à spire modérément élevée, formée de cinq tours et quart séparés par une suture très peu profonde, parfois presque invisible. Couleur blanc crème ornée de flammules orangées. Protoconque lisse d'un tour et quart environ; transition protoconque/téléoconque bien nette. Sculpture de la téléoconque constituée de côtes primaires fines croisées de macrostries spirales également fines mais bien marquées. Le réseau en résultant possède des mailles rectangulaires. Ombilic étroit, en forme de U, occupant 16,8 \% du diamètre au niveau du dernier tour. Ouverture en croissant de lune dévié par la carène; péristome légèrement prosocline, non continu, formant un angle légèrement évasé à la base du bord columellaire. Barrières aperturales: trois lamelles pariétales, la pariétale supérieure ( $\operatorname{Pr} 1)$ étant la plus forte; cinq lamelles palatales, la cinquième étant au dessus de la carène; pas de columellaire.

\section{DIMENSIONS}

Dimensions de l'holotype: $\mathrm{H}=1,70 \mathrm{~mm}$; $\mathrm{D}=$ $3,54 \mathrm{~mm} ; \mathrm{U}=0,60 \mathrm{~mm} ; \mathrm{L}=0,98 \mathrm{~mm} ; \mathrm{P}=$ $0,25 \mathrm{~mm} ; \mathrm{l}=1,35 \mathrm{~mm} ; \mathrm{h}=0,82 \mathrm{~mm} ; \mathrm{D} / \mathrm{U}=$ 5,$94 ; \mathrm{H} / \mathrm{D}=0,48 ; \mathrm{h} / \mathrm{l}=0,61$.

Dimensions moyennes et écarts-types pour 50 individus adultes (holotype, paratypes et matériel d'étude) : $\mathrm{H}=1,65 \mathrm{~mm} \pm 0,14 ; \mathrm{D}=3,33 \mathrm{~mm}$ $\pm 0,22 ; \mathrm{U}=0,56 \mathrm{~mm} \pm 0,06 ; \mathrm{L}=0,92 \mathrm{~mm} \pm 0,05$; $\mathrm{P}=0,30 \mathrm{~mm} \pm 0,07 ; \mathrm{l}=1,33 \mathrm{~mm} \pm 0,11 ; \mathrm{h}=$ $0,88 \mathrm{~mm} \pm 0,06 ; \mathrm{D} / \mathrm{U}=6,04 \pm 0,69 ; \mathrm{H} / \mathrm{D}=0,49$ $\pm 0,02 ; \mathrm{h} / \mathrm{l}=0,66 \pm 0,03$.

Les dimensions des paratypes sont données dans le Tableau 1.

\section{REMARQUES}

Le nombre des lamelles palatales est de quatre ou cinq selon les individus observés. Malgré beaucoup de similitudes, $A$. microspiralis n. sp. apparaît être une espèce distincte d'Australdonta tapina Solem, 1976 (Fig. 3E). La sculpture d'A. microspiralis n. sp. présente des stries spirales très nettes, visibles à l'oeil nu, ainsi qu'une suture peu marquée. Sa forme générale rappelle celle d'A. collicella, mais sa taille, la présence de côtes radiales visibles et la disposition des barrières aperturales rapprochent plus $A$. microspiralis n. sp. d'A. tapina et $A$. yoshii. Australdonta microspiralis n. sp. est toujours syntopique avec $A$. tapina.

\section{Australdonta sulcata n. sp.} (Figs 3D; 7)

Matériel type. - Holotype (MNHN 20958), 3 paratypes (MNHN 20959-20960), 1 paratype (BPBM 271803).

LOCAlité TYPE. - Rurutu, Mato Toarutu, versant ouest, sud de Pointe Mauo (sous grande grotte), pente rocheuse à Barringtonia et Pandanus, volcanique, $22,48062^{\circ} \mathrm{S}$, $151,3227^{\circ} \mathrm{W}, 15 \mathrm{~m}$, exposition E, 22.XI.2003, coll. Gargominy \& Fontaine, stn Rr39.

Étymologie. - Par ses côtes larges et espacées et la présence d'un sulcus, cette Australdonta ressemble à A. magnasulcata Solem, 1976, mais est légèrement plus petite et possède un sulcus moins marqué. Le nom sulcata (qui possède un sulcus) est de ce fait utilisé sans le préfixe latin magna- (grand).

MatéRIEl EXAminé. - Neuf spécimens, coll. Gargominy \& Fontaine dont holotype et 3 paratypes (MNHN), 1 paratype (BPBM).

Stn Rr20, Paparai, S Hauti, W Anatataura, bas de falaise calcaire, Musa, Macropiper, Aleurites, $22.50075^{\circ} \mathrm{S}$, $151.32813^{\circ} \mathrm{W}, 10 \mathrm{~m}$, exposition SW, 19.XI.2003, 2 spécimens. - Stn Rr22, falaise côtière calcaire, $S$ d'Hauti, N Pointe Poroa, pente rocheuse avec Pandanus, $22.49311^{\circ} \mathrm{S}$, $151.32392^{\circ} \mathrm{W}, 20 \mathrm{~m}$, exposition NE, 19.XI.2003, 3 spécimens (dont deux paratypes MNHN 20959). - Stn Rr39, 5 spécimens (dont holotype, 1 paratypes MNHN 20960 et 1 paratype BPBM 271803).

Distribution. - Endémique insulaire: Rurutu, entre la pointe Mauo et Anatataura, sur la côte est.

\section{DESCRIPTION (HOLOTYPE)}

Coquille fortement érodée, presque discoïde, à spire plane, formée de quatre tours et deux tiers séparés par une suture bien marquée. La spire porte un 


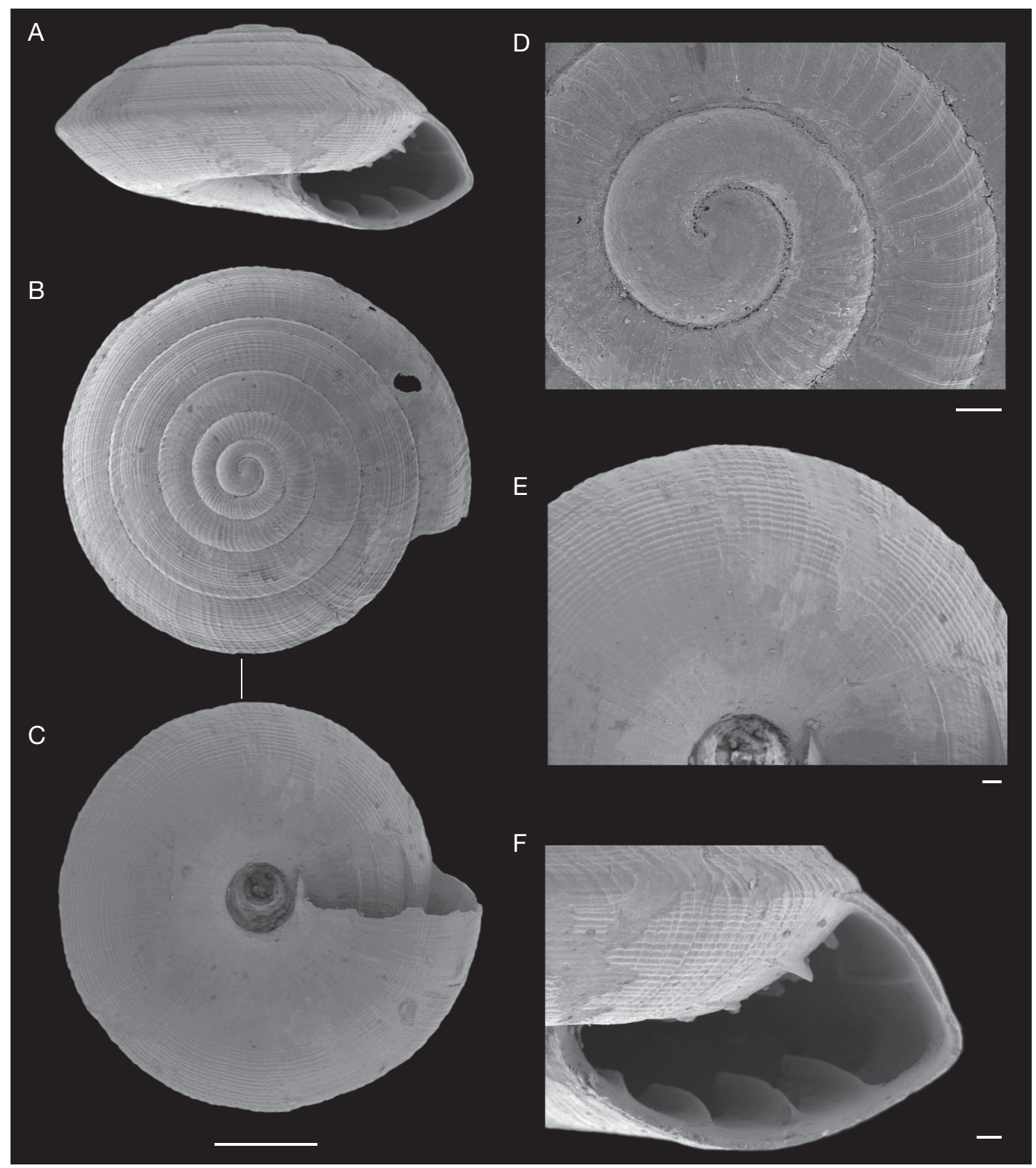

FIG. 6. - Australdonta microspiralis n. sp., Rurutu, îles Australes, Polynésie française (MEB): A, vue frontale; B, face supérieure; C, face inférieure; D, détail de la protoconque; E, sculpture du dernier tour, face inférieure; F, détail de l'ouverture. Échelles: A-C, 1 mm; $\mathrm{D}-\mathrm{F}, 100 \mu \mathrm{m}$.

bourrelet adapical proéminent. En vue frontale, la ligne médiane horizontale du dernier tour de spire est fortement inclinée. Protoconque également très érodée, de deux tours présumés, en apparence lisse, transition protoconque/téléoconque indistincte. Sculpture de la téléoconque constituée de côtes 


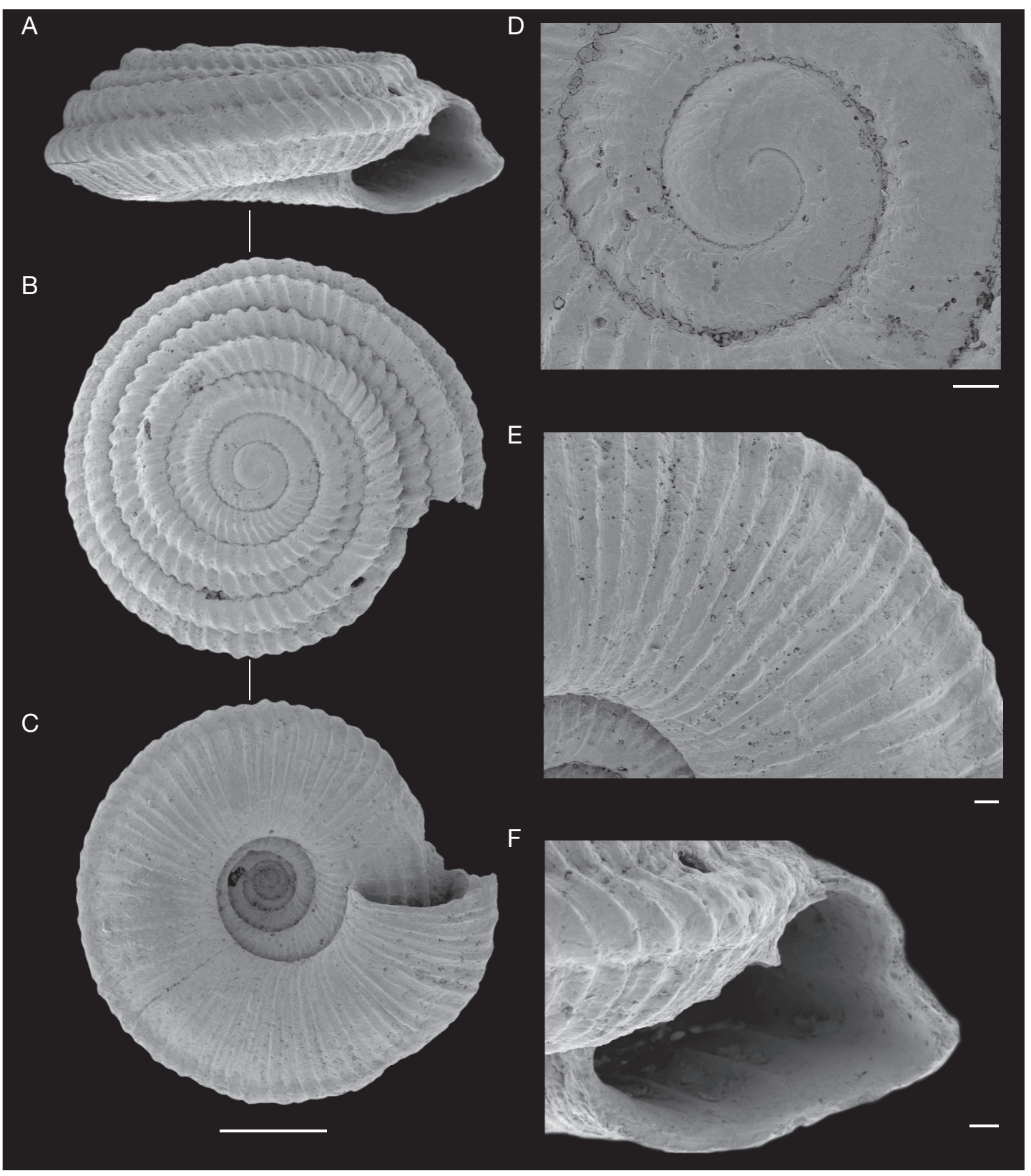

FIG. 7. - Australdonta sulcata n. sp., Rurutu, Îles Australes, Polynésie française (MEB): A, vue frontale; B, face supérieure; C, face inférieure; D, détail de la protoconque; E, sculpture du dernier tour, face inférieure; $\mathbf{F}$, détail de l'ouverture. Échelles: A-C, $1 \mathrm{~mm}$; $\mathrm{D}-\mathrm{F}, 100 \mu \mathrm{m}$.

primaires épaisses et modérément élevées, au nombre de 64 sur le dernier tour de spire, espacées de 160$170 \mu \mathrm{m}$. Microsculpture indiscernable en raison de l'érosion de la coquille. Ombilic en forme de $\mathrm{V}$ très évasé au niveau du dernier tour de la spire, occupant $34 \%$ du diamètre total. Ouverture en croissant 


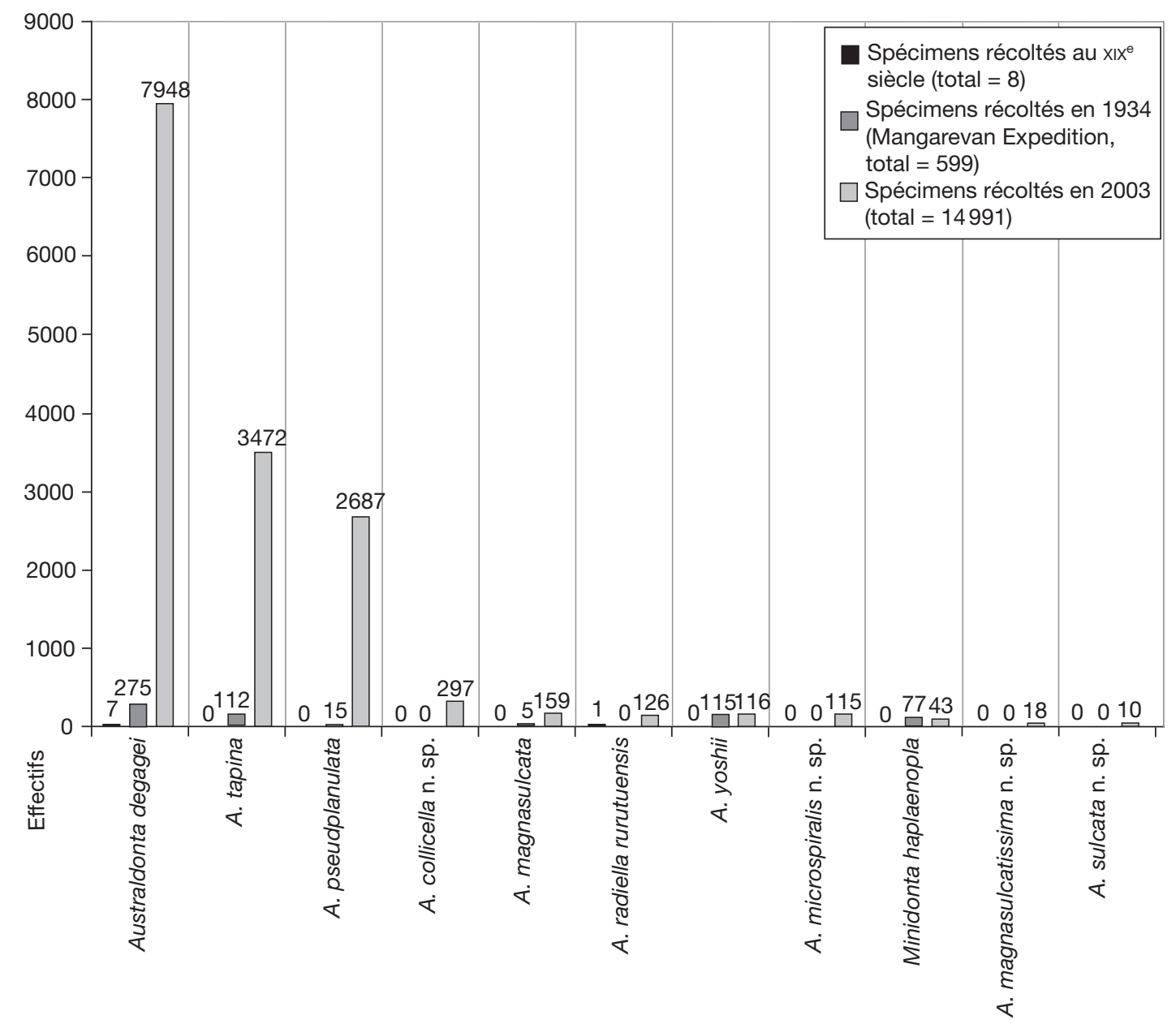

FIG. 8. - Nombre de spécimens d'Endodontidae récoltés pour chaque espèce au cours de l'expédition scientifique à Rurutu (2003).

de lune dévié par la carène; péristome légèrement prosocline, non continu, formant un angle évasé à la base du bord columellaire. Barrières aperturales: trois lamelles pariétales également espacées, la pariétale supérieure (Pr1) étant la plus forte; quatre lamelles palatales; pas de columellaire. Couleur blanc crème ornée de flammules orangées.

\section{DimEnSIONS}

Dimensions de l'holotype: $\mathrm{H}=1,21 \mathrm{~mm} ; \mathrm{D}=$ $3,59 \mathrm{~mm}$; $\mathrm{U}=1,23 \mathrm{~mm}$; $\mathrm{L}=0,90 \mathrm{~mm} ; \mathrm{P}=$ $0,00 \mathrm{~mm} ; \mathrm{l}=1,12 \mathrm{~mm} ; \mathrm{h}=0,86 \mathrm{~mm} ; \mathrm{D} / \mathrm{U}=$ 2,$91 ; \mathrm{H} / \mathrm{D}=0,34 ; \mathrm{h} / \mathrm{l}=0,77$.
Dimensions moyennes et écarts-types pour 9 individus adultes (holotype, paratypes 2 à 4 et matériel d'étude) : $\mathrm{H}=1,20 \mathrm{~mm} \pm 0,18 ; \mathrm{D}=3,44 \mathrm{~mm} \pm$ 0,$26 ; \mathrm{U}=1,13 \mathrm{~mm} \pm 0,15 ; \mathrm{L}=0,83 \mathrm{~mm} \pm 0,09$; $\mathrm{P}=0,00 \mathrm{~mm} \pm 0,00 ; \mathrm{l}=1,12 \mathrm{~mm} \pm 0,13 ; \mathrm{h}=$ $0,80 \mathrm{~mm} \pm 0,08 ; \mathrm{D} / \mathrm{U}=3,08 \pm 0,33 ; \mathrm{H} / \mathrm{D}=$ $0,35 \pm 0,04 ; \mathrm{h} / \mathrm{l}=0,72 \pm 0,04$.

Les dimensions des paratypes sont données dans le Tableau 1.

\section{REMARQUES}

Un des individus observés possède une quatrième lamelle pariétale. Australdonta sulcata n. sp. ressemble 
à Australdonta magnasulcata Solem, 1976, tout en étant plus petite, plane et carénée. La présence du bourrelet adapical est un caractère partagé par plusieurs espèces de Rurutu (A. magnasulcata, A. magnasulcatissima n. sp. et $A$. sulcata n. sp.), et est toujours accompagnée d'un épaississement des côtes. Australdonta sulcata n. sp. et A. magnasulcata ne sont syntopiques que pour la station $\operatorname{Rr} 20$.

\section{DISCUSSION ET CONCLUSIONS}

Le nombre d'espèces d'Endodontidae connus pour Rurutu est porté de sept à 11 . Les quatre espèces nouvelles décrites ici (Australdonta collicella n. sp., A. magnasulcatissima n. sp., A. microspiralis n. sp. et A. sulcata n. sp.) sont endémiques de l'île, et ont une distribution restreinte à la côte au vent, à l'est.

À l'exception d'A. collicella n. sp., ces espèces sont peu abondantes (Fig. 8), ce qui explique en partie qu'elles n'aient pas été récoltées par nos prédécesseurs. Le matériel examiné par Garrett (1879) ne comprenait que huit Endodontidae pour toute l'île de Rururu. La Mangarevan Expedition n'y récolta que 599 spécimens d'Endodontidae (Solem 1976), dans un nombre réduit de stations localisées dans les «matos» du nord, peut-être moins favorables à ces organismes.

Au cours des récoltes de 2003 et malgré les efforts de prospection mis en œuvre, seules des coquilles vides d'Endodontidae ont été récoltées, y compris dans les «matos», pourtant favorables au développement des gastéropodes terrestres. Aucun Endodontidae n'a été récolté vivant sur l'île depuis le XIXe siècle, la Mangarevan Expedition n'ayant rapporté que des coquilles vides (Cooke 1935; Solem 1976). La famille est très probablement éteinte sur Rurutu, même si il est possible que certaines espèces subsistent dans les anfractuosités profondes de la roche.

La disparition des Endodontidae de Rurutu illustre la grande vulnérabilité des espèces endémiques insulaires face aux facteurs anthropiques (MacArthur \& Wilson 1967). Le taux d'extinction de ces organismes est aujourd'hui alarmant (Bouchet \& Abdou 2003) et devrait entraîner des mesures de conservation des habitats, au même titre que pour la megafaune (Cowie 2001b). La forte érosion du couvert végétal par les activités agricoles (défrichages, brûlis, introduction d'herbivores) est à mettre en cause, et avait déjà été relatée par Cooke (1935) après la Mangarevan Expedition. En 2003 et pour une surface insulaire totale de 3230 hectares, nous n'avons relevé que 2 à 3 hectares de forêts naturelles humides d'altitude, dans des vallons difficilement accessibles à l'homme.

L'absence d'Endodontidae vivants sur Rurutu contraste avec l'abondance de certains Subulinidae allochtones. Un phénomène comparable a déjà été relevé pour l'archipel des Gambier (Abdou \& Bouchet 2000). Progressivement, une homogénéisation de la faune d'escargots terrestres semble s'imposer, à l'échelle de Rurutu et à l'échelle du Pacifique (Cowie 2001a, b).

Cette étude des Endodontidae de Rurutu pose le problème de la délimitation et de la caractérisation des espèces éteintes de Gastéropodes terrestres. Le travail de taxonomie a été contraint par l'absence de matériel vivant. Ce type de description, qui ne peut être fait sur des critères anatomiques et/ou moléculaires, est néanmoins nécessaire pour rendre compte de l'extraordinaire richesse passée de la faune de mollusques de Polynésie.

L'attribution d'un genre n'a pu être effectuée que sur la base des caractères conchyliologiques, à défaut de pouvoir observer les caractères anatomiques (concernant majoritairement l'appareil reproducteur) également utilisés par Solem (1976) pour définir les genres Australdonta et Minidonta.

La délimitation des espèces a également dû être établie sur la seule base des caractères conchyliologiques et n'a pas pu être confrontée à des résultats obtenus par des méthodes moléculaires.

Nous sommes aujourd'hui bien loin d'un bilan scientifique concernant les radiations et extinctions des escargots terrestres des îles du Pacifique. Il est nécessaire d'en nommer les espèces, éteintes ou non, pour qu'un tel bilan puisse être dressé et pour que des mesures de conservation des biotas favorables à ces organismes puissent être mises en œuvre.

\section{Remerciements}

Merci à Regina Kawamoto du B. P. Bishop Museum d'Honolulu pour le prêt des spécimens types de toutes les espèces d'Endodontidae des Australes. 
Gabrielle Zimmermann remercie Philippe Bouchet, Nadia Améziane, Jean-Michel Bichain et Marc Eléaume pour leur aide précieuse. Merci également à Michel Baylac et Raphaël Cornette, à la plateforme de morphométrie du MNHN.

Les illustrations au MEB ont été réalisées au service commun de microscopie électronique du MNHN, avec l'aide de Delphine Brabant et de Gérard Mascarell.

\section{RÉFÉRENCES}

ABDOU A. \& BoucheT P. 2000. — Nouveaux gastéropodes Endodontidae et Punctidae (Mollusca, Pulmonata) récemment éteints de l'archipel des Gambier (Polynésie). Zoosystema 22 (4): 689-707.

BAKer H. B. 1940. - Zonitid land snails from Pacific islands. Part 2: Hawaiian genera of Microcystinae. Bernice P. Bishop Museum Bulletin 165: 105-201, pls 21-42.

Bouchet P. \& ABdou A. 2003. - Endemic land snails from the Pacific islands and the museum record: Documenting and dating the extinction of the terrestrial Assimineidae of the Gambier Islands. Journal of Molluscan Studies 69: 165-170

Clouard V. \& Bonneville A. 2003. - Submarine landslides in Society and Austral Islands, French Polynesia: evolution with the age of edifices, in LOCAT J. \& MienerT J. (eds), Submarine Mass Movements and their Consequences. Kluwer Academic Publishers, Dordrecht: 335-341.

CoOKe C. M. 1935. - Report of C. Montague Cooke, Jr., malacologist and leader, Mangarevan Expedition. Bernice P. Bishop Museum Bulletin 133: 36-56.

COOKE C. M. \& KONDO Y. 1961. - Revision of Tornatellinidae and Achatinellidae (Gastropoda, Pulmonata). Bernice P. Bishop Museum Bulletin 221: 1-303.

COWIE R. H. 1992. - Evolution and extinction of Partulidae, endemic Pacific island land snails. Philosophical Transactions of the Royal Society of London, Series B, 335: 167-191.

COWIE R. H. 1996. - Pacific island land snails: relationships, origins, and determinants of diversity, in KEAST A. \& Miller S. E. (eds), The Origin and Evolution of Pacific Island Biotas, New Guinea to Eastern Polynesia: Patterns and Processes. SPB Academic Publishing, Amsterdam: 347-372.
CowIE R. H. 2001a. — Decline and homogenization of Pacific faunas: the land snails of American Samoa. Biological Conservation 99: 207-222.

CowIE R. H. 2001b. - Invertebrate invasions on Pacific Islands and the replacement of unique native faunas: a synthesis of the land and freshwater snails. Biological Invasions 3: 119-136.

GARRETT A. 1879. - List of land shells inhabiting Rurutu, one of the Austral Islands, with remarks on their synonymy, geographical range and descriptions of new species. Proceedings of the Academy of Natural Sciences of Philadelphia 31: 17-30.

Hadfield M. G. 1986. - Extinction in Hawaiian achantinelline snails. Malacologia 27: 67-81.

Hadfield M. G., Miller S. E. \& Carwile A. H. 1993. - The decimation of endemic Hawaiian tree snails by alien predators. American Zoologist 33: 610-622.

Hallé N. 1983. - Vegetation of Rurutu island; additions to the catalogue of the Southern islands flora. Bulletin du Muséum national d'Histoire naturelle, 4e sér., Section B, Adansonia 5 (2): 141-150.

Lydeard C., Cowie R. H., Ponder W. F., Bogan A. E., Bouchet P., Clark S., Cummings K. S., Frest T. J., Gargominy O., Herbert D. G., Hershler R., Perez K., Roth B., Seddon M., Strong E. E. \& Thompson F. G. 2004. - The global decline of nonmarine mollusks. BioScience 54: 321-330.

MacArthur R. \& Wilson E. O. 1967. - The Theory of Island Biogeography. Princeton University Press, Princeton, New Jersey, 203 p.

Pilsbry H. A. 1916. - Mid-Pacific land snail faunas. Proceedings of the National Academy of Sciences of the United States of America (2) 7: 429-433.

Preece R. C. 1998. - Impact of early Polynesian occupation on land snail fauna of Henderson Island, Pitcairn Group (South Pacific). Philosophical Transactions of the Royal Society of London B 353: 347-368.

Solem A. 1976. - Endodontoid Land Snails from Pacific Islands (Mollusca: Pulmonata: Sigmurethra). Part 1: Family Endodontidae. Field Museum of Natural History, Chicago, xii +508 p.

SolEM A. 1981. - Endodontoid Land Snails from Pacific Islands (Mollusca: Pulmonata: Sigmurethra). Part 2: Families Punctidae and Charopidae. Zoogeography. Field Museum of Natural History, Chicago, ix + 336 p.

Ohbayashi T., OkOchi I., Sato H., Ono T. \& Chiba S. 2007 - Rapid decline of endemic snails in the Ogasawara Islands, Western Pacific Ocean. Applied Entomology and Zoology 42: 479-485. 